\title{
Weak localization in ferromagnets with spin-orbit interaction
}

\author{
V. K. Dugaev ${ }^{1,2 \dagger}$, P. Bruno ${ }^{1}$, and J. Barnaśs S $^{3,4}$ \\ ${ }^{1}$ Max-Planck-Institut für Mikrostrukturphysik, Weinberg 2, D-06120 Halle, Germany \\ ${ }^{2}$ Institute of Materials Science Problems, Vilde 5, 58001 Chernovtsy, Ukraine \\ ${ }^{3}$ Department of Physics, A. Mickiewicz University, ul. Umultowska 85, 61-614 Poznań, Poland \\ ${ }^{4}$ Institute of Molecular Physics, Polish Academy of Sciences, \\ ul. M. Smoluchowskiego 17, 60-179 Poznań, Poland
}

(October 28, 2018)

\begin{abstract}
Weak localization corrections to conductivity of ferromagnetic systems are studied theoretically in the case when spin-orbit interaction plays a significant role. Two cases are analyzed in detail: (i) the case when the spin-orbit interaction is due to scattering from impurities, and (ii) the case when the spin-orbit interaction results from reduced dimensionality of the system and is of the Bychkov-Rashba type. Results of the analysis show that the localization corrections to conductivity of ferromagnetic metals lead to a negative magnetoresistance - also in the presence of the spin-orbit scattering. Positive magnetoresistance due to weak antilocalization, typical of nonmagnetic systems, does not occur in ferromagnetic systems. In the case of two-dimensional ferromagnets, the quantum corrections depend on the magnetization orientation with respect to the plane of the system.
\end{abstract}

PACS numbers: 72.25.-b, 72.15.Rn, 73.20.Fz

\section{INTRODUCTION}

Owing to the giant magnetoresistapce effect discovered in artificially layered structures, erties of low-dimensional magnetic systems were extensively studied in the past decade. The huge interest was stimulated by applications of the effect in magnetoelectronics, particularly in read/write heads, field sensors, random access memory elements, and others 3 Since the effect exists also at room temperatures (which is important for applications), there was only a little interest in the low temperature regime, where quantum corrections to conductivity may play a certain role. This regime, however, may be important in view of possible applications of metallic and/or semiconducting magnetic systems in spintronics and quantum computing

The quantum corrections to electrical conductivity, related to scattering of electrons from impurities in nonmagnetic metals and doped semiconducters, were extensively studied in the past two decades. 0 However, the problem of quantum corrections in ferromagnetic metals is still unexplored. Only a few works on this subject can be found in the refeyant literature. These include two theoretical work1 19,11 and a few reports on experiments 1217 which prove the existence and importance of the quantum corrections related to both weak localization and electron-electron interaction effects. The theoretical description, however, was restricted to the effects of localization on the spin-density fluctuations in the vicinity of the ferromagnetic transition 10 and to electronelectron interaction effects in spin dependent quantum wells 11 .

It is well known that the quantum corrections due to weak localization in nonmagnetic systems are suppressed by a sufficiently large magnetic induction $\mathbf{B}$. One may then expect a similar suppression of weak localization by an internal magnetic induction $B_{\text {int }}$ in ferromagnets. But this point is still not definitely clear from the experimental point of view, at least for some kinds of ferromagnetic materials. It is then reasonable to assume that the internal magnetic induction existing inside the ferromagnets may reduce the localization corrections instead of destroying them totally. Very likely, one can expect only a slight effect of $B_{\text {int }}$ in the case of newlydeveloped magnetic semiconductors like GaMnAs alloys 18

Spin-orbit (SO) scattering from paramagnetic impurities in nonmagnetic metals is known to have a significant influence on the quantum corrections. It can reverse the sign of the localization correction (so-called weak antilocalization effect), which results in a positive magnetoresistance at weak magnetic fields 1921 However, SO interaction may also result from other sources like for example the Dresselhau 22 or Bychkov-Rashba23 terms in the relevant Hamiltonian. These terms are related to the lack of inversion symmetry in certain crystals or to reduced dimensionality in quantum-confined structures, respectively. In the context of weak localization theory, this type of spin-orbit interaction has beanstudied by A. G. Aronov and Yu. B. Lyanda-Geller.24 26

Recently, there is a large interest in SO interaction in magnetically ordered materials. First, the SO scattering is believed to be responsible for the anomalous Hall effect in ferromagnets.27 29 Second, the $\mathrm{SO}$ interaction is one of the main interactions which determine the spin diffusion length. The latter quantity plays a crucial role in the dependence of the giant magnetoresistance effect on the sublayer thicknesse, when the current flows perpendicularly to the films.30]31

In this paper we study the localization corrections to conductivity of ferromagnetic systems in the presence 
of SO scattering from defects and also in the presence of the Bychkov-Rashba term. It is well known that in nonmagnetic materials the spin-orbit scattering is crucial for the localization correction. As it was already mentioned above, the SO scattering leads to weak antilocalization, i to positive magnetoresistance at small magnetic fields.19 21 The situation in ferromagnetic metals, however, is significantly different. We show that the processes, leading to weak antilocalization in nonmagnetic systems, are totally suppressed in ferromagnets, so in the presence of SO interaction we have only a negative magnetoresistance.

In Sections 2 and 3 we derive the formula for the Cooperon and quantum correction to conductivity in three-dimensional (3D) ferromagnets. Two dimensional (2D) ferromagnets are considered in Section 4, while quantum wells and possible crossover from $2 \mathrm{D}$ to $3 \mathrm{D}$ case are discussed in Section 5. The influence of SO interaction in the form of Bychkov-Rashba term is studied in Section 6. In Section 7 we discuss the effect of internal magnetization on the localization corrections. Finally, conclusions and summary are in Section 8.

\section{COOPERON IN A 3D FERROMAGNET}

We consider the following model Hamiltonian of a ferromagnet with SO scattering:

$$
H=\int d^{3} \mathbf{r} \psi^{\dagger}(\mathbf{r})\left[-\frac{\nabla^{2}}{2 m}-M \sigma_{z}+V(\mathbf{r})\right] \psi(\mathbf{r})
$$

where the axis $z$ is assumed to be along the magnetization $\mathbf{M}, \psi$ is a spinor field with the components $\psi_{\uparrow}$ and $\psi_{\downarrow}$, and we put $\hbar=1$. In the presence of a magnetic induction $\mathbf{B}=\operatorname{rot} \mathbf{A}$, the gradient operator $\nabla$ is replaced by $\nabla-i e \mathbf{A} / c$. Note that the exchange term $-M \sigma_{z}$ acts only on the spin and has therefore no direct effect on the orbital motion of the electrons.

The random potential $V(\mathbf{r})$ of impurities consists of two statistically independent components - the component independent of the electron spin, described by the random potential $V_{0}(\mathbf{r})$, and the spin-orbit component $V_{\text {so }}(\mathbf{r})$. Matrix elements of the latter component have the form

$$
\left(V_{s o}\right)_{\mathbf{k} \alpha, \mathbf{k}^{\prime} \beta}=i V_{1}\left(\mathbf{k} \times \mathbf{k}^{\prime}\right) \cdot \sigma_{\alpha \beta}
$$

for the transitions $(\mathbf{k}, \alpha) \rightarrow\left(\mathbf{k}^{\prime}, \beta\right)$, where $V_{1}$ is a constant, $\mathbf{k}$ and $\mathbf{k}^{\prime}$ are the initial and final electron wavevectors, $\alpha$ and $\beta$ describe the corresponding spin states, and $\sigma=\left(\sigma_{x}, \sigma_{y}, \sigma_{z}\right)$ are the Pauli matrices.

The key component of the weak localization theory is the Cooperon, 9 which can be presented by a ladder in the particle-particle channel with two propagators describing electrons with almost vanishing total momentum and with very close energy parameters (so-called Cooper channel). In the case of ferromagnets, and as long as
$M \gg \tau_{\uparrow}^{-1}, \tau_{\downarrow}^{-1}$, where $\tau_{\uparrow}$ and $\tau_{\downarrow}$ are the momentum relaxation times of the spin up and down electrons at the Fermi surface, this channel does not include ladder elements with Green functions corresponding to the opposite spin orientations. Indeed, using the standard method of calculation of the Cooperon, 6 one should evaluate the integral

$$
\Pi_{\sigma \sigma^{\prime}}=\int \frac{d^{3} \mathbf{k}}{(2 \pi)^{3}} G_{\sigma}^{R}(\varepsilon, \mathbf{k}) G_{\sigma^{\prime}}^{A}(\varepsilon,-\mathbf{k}),
$$

where $G_{\sigma}^{R, A}(\varepsilon, \mathbf{k})$ are the retarded and advanced Green functions of an electron with spin $\sigma=\uparrow, \downarrow$. This gives $\Pi_{\uparrow \downarrow} / \Pi_{\uparrow \uparrow} \simeq 1 / M \tau_{\uparrow} \ll 1$, and $\Pi_{\uparrow \downarrow} / \Pi_{\downarrow \downarrow} \simeq 1 / M \tau_{\downarrow} \ll 1$, which corresponds to vanishingly small contribution of the singlet channel. This result can also be understood as a suppression of singlet pairs by the exchange field.

Validity of this approach is confirmed by the following estimations. If we assume parameters typical for pure Fe, 32 i.e., $M=2.5 \mathrm{eV}$ and $\tau_{\uparrow} \simeq \tau_{\downarrow} \simeq 5 \times 10^{-13} \mathrm{~s}$, we obtain $\left(M \tau_{\uparrow, \downarrow}\right)^{-1} \simeq 5 \times 10^{-4}$. In dirty $\mathrm{Fe}$, this value would be increased by one or two orders of magnitude, but will still remain small as compare to unity. The exclusion of the Cooperon in the singlet channel is the crucial point of our description, which leads to the absence of weak antilocalization in ferromagnets. In the following, we will omit the spin index in the inequalities $M \gg \tau_{\uparrow}^{-1}, \tau_{\downarrow}^{-1}$, and will write simply $M \gg \tau^{-1}$.

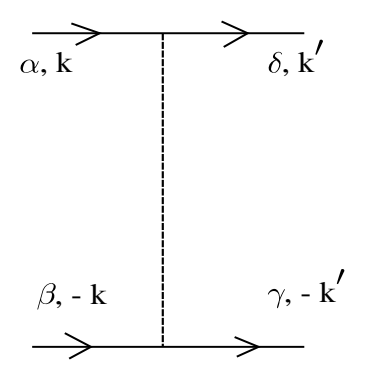

FIG. 1. The bare scattering amplitude.

In the case of weak scattering potential and upon averaging over the random field $V(\mathbf{r})$, one finds the following bare scattering amplitude (see Fig. 1):

$$
\Gamma_{\alpha \beta \gamma \delta}^{0}=a \delta_{\alpha \delta} \delta_{\beta \gamma}-b\left(\sigma_{\alpha \delta}^{x} \sigma_{\beta \gamma}^{x}+\sigma_{\alpha \delta}^{y} \sigma_{\beta \gamma}^{y}\right)-t_{\alpha \beta \gamma \delta},
$$

where $t_{\alpha \beta \gamma \delta}$ is a diagonal matrix in the space of $(\uparrow \uparrow),(\uparrow \downarrow$ )$,(\downarrow \uparrow),(\downarrow \downarrow)$ states,

$$
\mathbf{t}=\operatorname{diag}\left(d_{1}, d_{3}, d_{3}, d_{2}\right),
$$

and we introduced the following definitions:

$$
\begin{gathered}
a=N_{i} V_{0}^{2}=\frac{1}{2 \pi \nu_{\uparrow} \tau_{0 \uparrow}}=\frac{1}{2 \pi \nu_{\downarrow} \tau_{0 \downarrow}}, \\
b=N_{i} V_{1}^{2} \lambda_{0} k_{F \uparrow}^{2} k_{F \downarrow}^{2},
\end{gathered}
$$




$$
\begin{gathered}
d_{1}=N_{i} V_{1}^{2} \lambda_{0} k_{F \uparrow}^{4}=\frac{1}{2 \pi \nu_{\uparrow} \tau_{s o \uparrow}^{z}}, \\
d_{2}=N_{i} V_{1}^{2} \lambda_{0} k_{F \downarrow}^{4}=\frac{1}{2 \pi \nu_{\downarrow} \tau_{s o \downarrow}^{z}}, \\
d_{3}=-N_{i} V_{1}^{2} \lambda_{0} k_{F \uparrow}^{2} k_{F \downarrow}^{2},
\end{gathered}
$$

with $\lambda_{0}$ defined as

$$
\lambda_{0}=\overline{\left(\mathbf{n}_{\mathbf{k}} \times \mathbf{n}_{\mathbf{k}^{\prime}}\right)_{x}^{2}}=\frac{2}{9} .
$$

In the above equations $N_{i}$ is the concentration of scattering centers, $\nu_{\uparrow}$ and $\nu_{\downarrow}$ are the densities of states at the Fermi level for spin-up (majority) and spin-down (minority) electrons, while $k_{F \uparrow}$ and $k_{F \downarrow}$ are the Fermi wavevectors for spin-up and spin-down electrons. The relaxation times $\tau_{0 \uparrow}$ and $\tau_{0 \downarrow}$, defined by Eq. (5), are the momentum relaxation times in the absence of SO scattering, whereas the relaxation times $\tau_{\text {so }}^{z}$ and $\tau_{s o \downarrow}^{z}$, defined by Eqs. (7) and (8), are due to the SO scattering. The averaging in Eq. (10) is over all orientations of the unit vectors $\mathbf{n}_{\mathbf{k}}$ and $\mathbf{n}_{\mathbf{k}^{\prime}}$, where $\mathbf{n}_{\mathbf{k}}=\mathbf{k} / k$ and $\mathbf{n}_{\mathbf{k}^{\prime}}=\mathbf{k}^{\prime} / k^{\prime}$.

Using Eq. (3), we find the following bare scattering amplitudes for the spin-up and spin-down electrons:

$$
\begin{gathered}
\Gamma_{\uparrow}^{0} \equiv \Gamma_{\uparrow \uparrow \uparrow \uparrow}^{0}=a-d_{1}=\frac{1}{2 \pi \nu_{\uparrow} \tau_{0 \uparrow}}-\frac{1}{2 \pi \nu_{\uparrow} \tau_{s o \uparrow}^{z}}, \\
\Gamma_{\downarrow}^{0} \equiv \Gamma_{\downarrow \downarrow \downarrow \downarrow}^{0}=a-d_{2}=\frac{1}{2 \pi \nu_{\downarrow} \tau_{0 \downarrow}}-\frac{1}{2 \pi \nu_{\downarrow} \tau_{s o \downarrow}^{z}} .
\end{gathered}
$$

One should note at this point that the bare elements $\Gamma_{\uparrow \downarrow \uparrow \downarrow}^{0}$ and $\Gamma_{\downarrow \uparrow \downarrow \uparrow \uparrow}^{0}$ do not contribute to the Cooper-channel diagrams in the case of ferromagnetic systems as long as $M>>1 / \tau$, as we already stated before. Apart from this, in a $3 \mathrm{D}$ case $\Gamma^{0}$ does not contain the components $\Gamma_{\uparrow \uparrow \downarrow \downarrow}^{0}$ and $\Gamma_{\downarrow \downarrow \uparrow \uparrow}^{0}$, which vanish due to the rotational symmetry in the $x-y$ plane, as can also be concluded from Eq. (3).

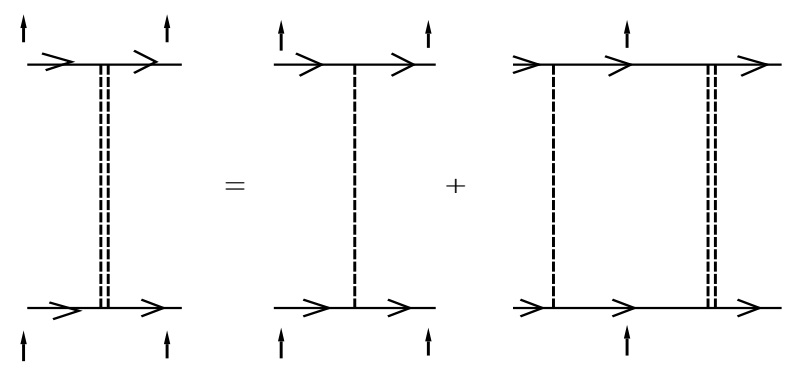

FIG. 2. Ladder diagram for the Cooperon in 3D case.
Summing up the ladder diagrams contributing to the renormalized scattering amplitude $\Gamma_{\uparrow}(\omega, q) \equiv \Gamma_{\uparrow \uparrow \uparrow \uparrow}(\omega, q)$ in the Cooper channel with small transferred energy $\omega$ and momentum $q$ 6. (see Fig. 2), one obtains the equation

$$
\Gamma_{\uparrow}(\omega, q)=\Gamma_{\uparrow}^{0}+\Gamma_{\uparrow}^{0} \Pi_{\uparrow}(\omega, q) \Gamma_{\uparrow}(\omega, q),
$$

where

$$
\begin{gathered}
\Pi_{\uparrow}(\omega, q)=\int \frac{d^{3} \mathbf{k}}{(2 \pi)^{3}} G_{\uparrow}^{R}(\omega, \mathbf{k}+\mathbf{q}) G_{\uparrow}^{A}(0,-\mathbf{k}) \\
\simeq 2 \pi \nu_{\uparrow} \tau_{\uparrow}\left(1-D_{\uparrow} q^{2} \tau_{\uparrow}+i \omega \tau_{\uparrow}\right) .
\end{gathered}
$$

Here, $D_{\uparrow}=\frac{1}{3} v_{F \uparrow}^{2} \tau_{\uparrow}$ is the diffusion coefficient, $v_{F \uparrow}$ is the Fermi velocity, and $\tau_{\uparrow}$ is the momentum relaxation time of spin up electrons. Equation (14) was derived in the diffusion limit, i.e., when $\omega \ll 1 / \tau_{\uparrow}$ and $q \ll\left(D_{\uparrow} \tau_{\uparrow}\right)^{-1 / 2}$.
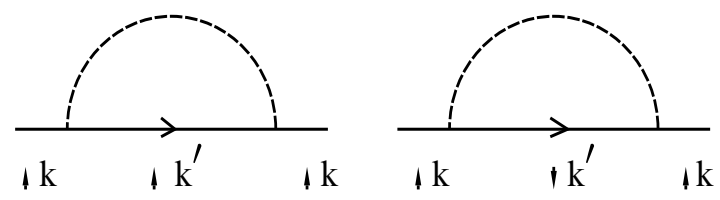

FIG. 3. Self energy diagram.

The relaxation time $\tau_{\uparrow}$ can be found by calculating the self energy, presented by the diagrams of Fig. 3. The self energy contains non-zero spin-flip vertices of the singlet type, as shown in Fig. 4. After calculating the imaginary part of the self energy, we find

$$
\frac{1}{\tau_{\uparrow}}=2 \pi \nu_{\uparrow}\left(a+d_{1}+\frac{2 \nu_{\downarrow}}{\nu_{\uparrow}} b\right) .
$$

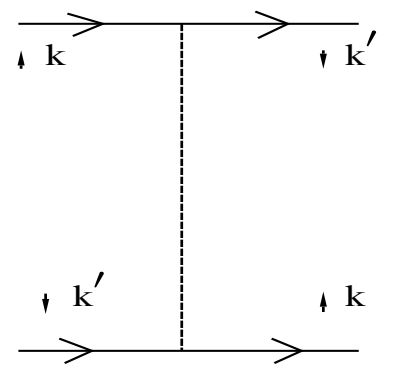

FIG. 4. Spin-flip vertex. 
Using Eqs. (5) to (14), we finally obtain the following formula for the renormalized scattering amplitude in the Cooper channel (Cooperon):

$$
\Gamma_{\uparrow}(\omega, q)=\frac{1}{\tau_{\uparrow}} \frac{a+d_{1}+\left(2 \nu_{\downarrow} / \nu_{\uparrow}\right) b}{-i \omega+D_{\uparrow} q^{2}+1 / \tilde{\tau}_{s o \uparrow}},
$$

where the effective SO relaxation time of Cooperon, $\tilde{\tau}_{s o \uparrow}$, is introduced,

$$
\frac{1}{\tilde{\tau}_{s o \uparrow}}=\frac{2}{\tau_{\uparrow}} \frac{d_{1}+\left(\nu_{\downarrow} / \nu_{\uparrow}\right) b}{a-d_{1}} .
$$

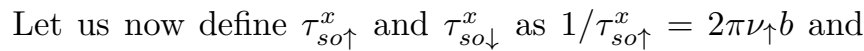
$1 / \tau_{\text {so } \downarrow}^{x}=2 \pi \nu_{\downarrow} b$. In the limit of weak SO scattering, $\tau_{\text {so }}^{z} \gg \tau_{\uparrow}$, one may then write

$$
\Gamma_{\uparrow}(\omega, q)=\frac{1}{2 \pi \nu_{\uparrow} \tau_{\uparrow}^{2}} \frac{1}{-i \omega+D_{\uparrow} q^{2}+1 / \tilde{\tau}_{s o \uparrow}+1 / \tau_{\varphi \uparrow}},
$$

where

$$
\frac{1}{\tilde{\tau}_{s o \uparrow}}=2\left(\frac{1}{\tau_{s o \uparrow}^{z}}+\frac{\nu_{\downarrow}}{\nu_{\uparrow}} \frac{1}{\tau_{s o \uparrow}^{x}}\right),
$$

and a phase relaxation time $\tau_{\varphi \uparrow}$, related to some inelastic scattering processes of electrons, 6 is added into Eq. (18).

The analogous formulae can also be derived for the spin-down Cooperon $\Gamma_{\downarrow}(\omega, q) \equiv \Gamma_{\downarrow \downarrow \downarrow \downarrow}(\omega, q)$. This formula can be obtained from Eqs. (18) and (19) by inverting direction of the arrows.

\section{CONDUCTIVITY AND MAGNETOCONDUCTIVITY IN 3D CASE}

The localization correction $\Delta \sigma$ tothe static conductivity is determined by the diagrams 1 :
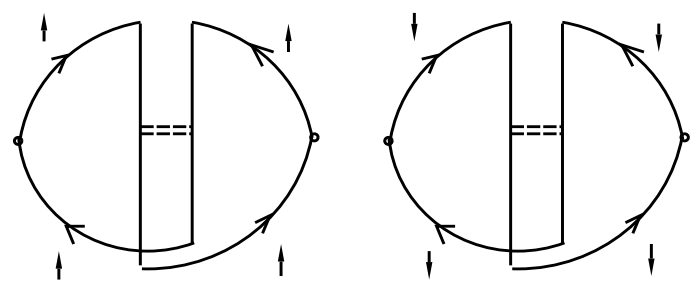

FIG. 5. Localization corrections to conductivity.

Upon calculating the corresponding contributions, one finds the following formula for $\Delta \sigma$ :

$$
\Delta \sigma=-\frac{e^{2}}{\pi}\left(2 \pi \nu_{\uparrow} \tau_{\uparrow}^{2} D_{\uparrow} \int \frac{d^{3} \mathbf{q}}{(2 \pi)^{3}} \Gamma_{\uparrow}(0, q)+2 \pi \nu_{\downarrow} \tau_{\downarrow}^{2} D_{\downarrow} \int \frac{d^{3} \mathbf{q}}{(2 \pi)^{3}} 2 \pi \nu_{\downarrow} \Gamma_{\downarrow}(0, q)\right),
$$

which is a straightforward generalization of the localization correction in a nonmagnetic case. Using Eqs. (18) and (20), we obtain

$$
\Delta \sigma=\text { const }+\frac{e^{2}}{4 \pi^{2}}\left[\frac{1}{D_{\uparrow}^{1 / 2}}\left(\frac{1}{\tilde{\tau}_{s o \uparrow}}+\frac{1}{\tau_{\varphi \uparrow}}\right)^{1 / 2}+\frac{1}{D_{\downarrow}^{1 / 2}}\left(\frac{1}{\tilde{\tau}_{s o \downarrow}}+\frac{1}{\tau_{\varphi \downarrow}}\right)^{1 / 2}\right] .
$$

where the constant part is related to the contribution from the largest momenta of Cooperon, $q \sim(D \tau)^{-1 / 2}$, and can not be calculated exactly within the diffusion approximation.68 8 It can be estimated as

$$
\text { const } \simeq-\frac{e^{2}}{4 \pi^{2}}\left[\left(D_{\uparrow} \tau_{\uparrow}\right)^{-1 / 2}+\left(D_{\downarrow} \tau_{\downarrow}\right)^{-1 / 2}\right] .
$$

Thus, the constant term is negative and, since $\tau_{\uparrow, \downarrow}<\tilde{\tau}_{s o}, \tau_{\varphi}$, it is larger in magnitude than the second term of Eq. (21). Therefore, the total correction (21) is negative. By decreasing $\tilde{\tau}_{s o}$ and/or $\tau_{\varphi}$, we suppress the localization correction to conductivity.

The magnetic induction (both external and internal) supresses the localization corrections. If the magnitude of the total magnetic induction is $B$, then, following the method developed by Kawabata, 33 we find

$$
\begin{gathered}
\Delta \sigma(B)=-\frac{e^{2}}{\pi}\left(D_{\uparrow} \frac{e B}{2 \pi c} \sum_{n=0}^{n_{0 \uparrow}} \int_{-\infty}^{\infty} \frac{d q}{2 \pi}\left[D_{\uparrow} q^{2}+\frac{4 e B D_{\uparrow}}{c}\left(n+\frac{1}{2}\right)+\frac{1}{\tilde{\tau}_{s o \uparrow}}+\frac{1}{\tau_{\varphi \uparrow}}\right]^{-1}\right. \\
\left.+D_{\downarrow} \frac{e B}{2 \pi c} \sum_{n=0}^{n_{0 \downarrow}} \int_{-\infty}^{\infty} \frac{d q}{2 \pi}\left[D_{\downarrow} q^{2}+\frac{4 e B D_{\downarrow}}{c}\left(n+\frac{1}{2}\right)+\frac{1}{\tilde{\tau}_{s o \downarrow}}+\frac{1}{\tau_{\varphi \downarrow}}\right]^{-1}\right),
\end{gathered}
$$


where $l_{B}=(c / e B)^{1 / 2}$ is the magnetic length, $c$ is the light velocity, and the sums over the Landau levels are cut off at $n_{0 \uparrow(\downarrow)} \simeq l_{B}^{2} /\left(D_{\uparrow(\downarrow)} \tau_{\uparrow(\downarrow)}\right)$.

After eliminating the $\Delta \sigma(B=0)$ part, 33 we find a formula, which is a generalization of the Kawabata's low-field magnetoresistance to the ferromagnetic case,

$$
\begin{gathered}
\Delta \sigma(B)-\Delta \sigma(0)=-\frac{e^{2}}{16 \pi^{2} l_{B}} \sum_{n=0}^{\infty}\left[\frac{1}{\left(n+1 / 2+\delta_{\uparrow}\right)^{1 / 2}}-2\left(n+1+\delta_{\uparrow}\right)^{1 / 2}+2\left(n+\delta_{\uparrow}\right)^{1 / 2}\right. \\
\left.+\frac{1}{\left(n+1 / 2+\delta_{\downarrow}\right)^{1 / 2}}-2\left(n+1+\delta_{\downarrow}\right)^{1 / 2}+2\left(n+\delta_{\downarrow}\right)^{1 / 2}\right],
\end{gathered}
$$

where

$$
\delta_{\uparrow(\downarrow)}=\frac{l_{B}^{2}}{4 D_{\uparrow(\downarrow)}}\left(\frac{1}{\tilde{\tau}_{s o \uparrow(\downarrow)}}+\frac{1}{\tau_{\varphi \uparrow(\downarrow)}}\right) .
$$

In accordance with Eq. (22), the magnetic induction supresses the negative correction to the conductivity. Thus, the resulting sign of Eq. (23) is positive, and its magnitude, $\Delta \sigma(B)-\Delta \sigma(0)$, increases with increasing magnetic field. This means that one finds a negative magnetoresistance, despite of the presence of the spin-orbit interaction. The reason of this is the fact that we have excluded the singlet Cooperon, which contributes to the localization correction with the opposite sign, and usually gives rise to a positive magnetoresistance (weak antilocalizating in weak magnetic fields in nonmagnetic materials.6.

The obtained result is in agreement with the results on magnetoconductivity of nonmagnetic metals in a magnetic field, if both Zeeman splitting and spin-orbit scattering are taken into account.21 1 Indeed, the exchange field of a ferromagnet enters the Hamiltonian, Eq. (1), like the Zeeman term. Thus, the strong exchange-field limit of a ferromagnet corresponds to the case of a large magnetic field in the Zeeman term. At these conditions, the effect of a magnetic field is associated with a negative magnetoresistance due to the suppression of the singlet Cooperon by magnetic field through the Zeeman splitting.

\section{TWO-DIMENSIONAL FERROMAGNETS}

In this section we consider a two-dimensional ferromagnet. In such a case there is no electron motion in the direction perpendicular to the plane, and consequently the electron wavevectors are in the plane of the ferromagnet. We consider first the case of in-plane magnetization, as shown schematically in Fig. 6.

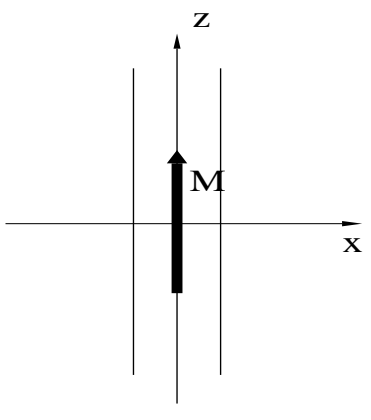

FIG. 6. 2D ferromagnet with in-plane magnetization.

The bare scattering amplitude has then the form

$$
\Gamma_{\alpha \beta \gamma \delta}^{0}=a \delta_{\alpha \delta} \delta_{\beta \gamma}-b \sigma_{\alpha \delta}^{x} \sigma_{\beta \gamma}^{x},
$$

where

$$
a=\frac{1}{2 \pi \nu \tau_{0}}
$$

and

$$
b=\frac{1}{2 \pi \nu \tau_{s o}^{x}} .
$$

In a strictly 2D case and when both spin sub-bands are populated with electrons, the density of states is independent of the spin orientation, $\nu_{\uparrow}=\nu_{\downarrow} \equiv \nu$. This follows from the model assumed in Eq. (2). In that case also the relaxation times in the absence and/or presence of the SO scattering are independent of the electron spin,

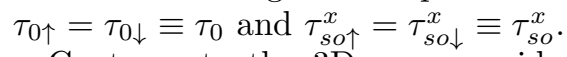

Contrary to the $3 \mathrm{D}$ case considered above, we have now to include the spin-flip processes in the Cooperon ladder. This is due to the fact that now we do not have the rotational symmetry in the $x-y$ plane. According to Eqs. (25) to (27), the bare amplitudes are now,

$$
\begin{gathered}
\Gamma_{\uparrow}^{0}=\Gamma_{\downarrow}^{0} \equiv \Gamma^{0}=\frac{1}{2 \pi \nu \tau_{0}}, \\
\Gamma_{\uparrow \uparrow \downarrow \downarrow}^{0}=\Gamma_{\downarrow \downarrow \uparrow \uparrow}^{0} \equiv \Gamma_{s f}^{0}=-\frac{1}{2 \pi \nu \tau_{s o}^{x}} .
\end{gathered}
$$


The equations for the renormalized vertices can be written as two coupled ladders for $\Gamma_{\uparrow \uparrow \uparrow \uparrow}(\omega, q)=\Gamma_{\downarrow \downarrow \downarrow \downarrow}(\omega, q) \equiv$ $\Gamma(\omega, q)$ and $\Gamma_{\uparrow \uparrow \downarrow \downarrow}(\omega, q)=\Gamma_{\downarrow \downarrow \uparrow \uparrow}(\omega, q) \equiv \Gamma_{s f}(\omega, q)$, as shown in Fig. 7.
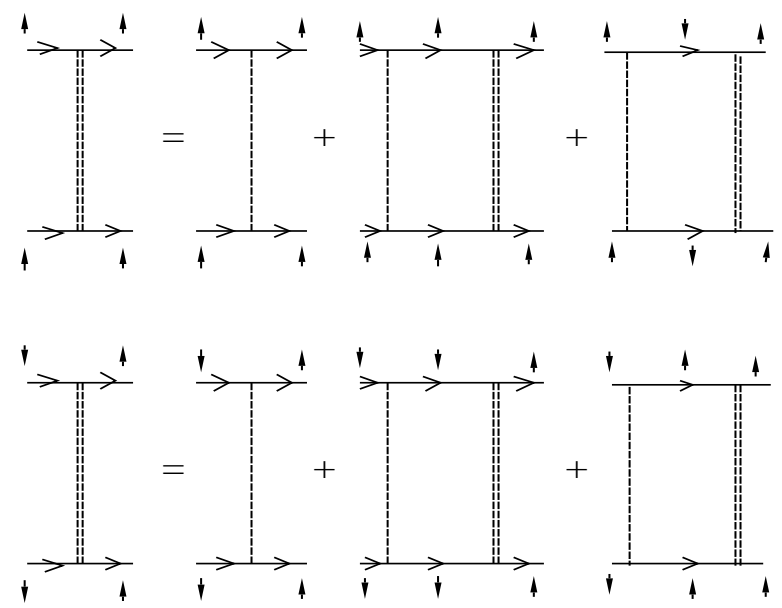

FIG. 7. Equation for the Cooperon in 2D ferromagnet with in-plane magnetization.

From these equations we find

$$
\Gamma(\omega, q)=\frac{1}{4 \pi \nu \tau^{2}} \frac{1}{-i \omega+\bar{D} q^{2}+1 / \tau_{\varphi}}
$$

where $\bar{D}=\frac{1}{2}\left(D_{\uparrow}+D_{\downarrow}\right)$, and the diffusion constants $D_{\uparrow}$ and $D_{\downarrow}$ are defined as $D_{\uparrow(\downarrow)}=\frac{1}{2} v_{F \uparrow(\downarrow)}^{2} \tau$. The electron relaxation time $\tau$ in Eq. (30) is independent of the electron spin, $\tau_{\uparrow}=\tau_{\downarrow} \equiv \tau$, and can be calculated in the same way as in the $3 \mathrm{D}$ case, which gives

$$
\frac{1}{\tau_{\uparrow}}=\frac{1}{\tau_{\downarrow}} \equiv \frac{1}{\tau}=\frac{1}{\tau_{0}}+\frac{1}{\tau_{\text {so }}^{x}}
$$

It is worth to note that the spin-orbit scattering enters the Cooperon only through the one-particle relaxation time $\tau$, and has no influence on the pole of the Cooperon.

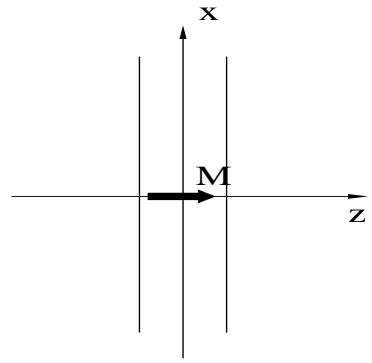

FIG. 8. 2D ferromagnet with magnetization perpendicular to its plane.

Consider now the case when the magnetization field $\mathbf{M}$ is perpendicular to the plane of the ferromagnet, as shown schematically in Fig. 8. The calculations are similar to those in the case of in-plane magnetization, so we write down only the results. The only bare scattering amplitudes are now $\Gamma_{\uparrow}^{0}$ and $\Gamma_{\downarrow}^{0}$, which generally are different and have the form

$$
\Gamma_{\uparrow(\downarrow)}^{0}=\frac{1}{2 \pi \nu \tau_{0}}-\frac{1}{2 \pi \nu \tau_{s o \uparrow(\downarrow)}^{z}} .
$$

The Cooperons $\Gamma_{\uparrow}(\omega, q)$ and $\Gamma_{\downarrow}(\omega, q)$ can be written as

$\Gamma_{\uparrow(\downarrow)}(\omega, q)=\frac{1}{2 \pi \nu \tau_{\uparrow(\downarrow)}^{2}} \frac{1}{-i \omega+D_{\uparrow(\downarrow)} q^{2}+1 / \tilde{\tau}_{s o \uparrow(\downarrow)}+1 / \tau_{\varphi \uparrow(\downarrow)}}$,

where the relaxation times $\tau_{\uparrow}$ and $\tau_{\downarrow}$ are given by

$$
\frac{1}{\tau_{\uparrow(\downarrow)}}=\frac{1}{\tau_{0}}+\frac{1}{\tau_{\text {so } \uparrow(\downarrow)}^{z}},
$$

and

$$
\frac{1}{\tilde{\tau}_{s o \uparrow(\downarrow)}}=\frac{2}{\tau_{s o \uparrow(\downarrow)}^{z}-2 \tau_{\uparrow(\downarrow)}} \simeq \frac{2}{\tau_{s o \uparrow(\downarrow)}^{z}},
$$

provided that $\tau_{\text {so } \uparrow(\downarrow)}^{z} \gg \tau_{\uparrow(\downarrow)}$. By comparing the results (30) and (33), both obtained for a 2D case but for different magnetic configurations, we see that the effect of SO interaction significantly depends on the orientation of the magnetization field $\mathbf{M}$ with respect to the plane of the ferromagnet.

The quantum correction to the conductivity of a $2 \mathrm{D}$ ferromagnet takes the form

$$
\Delta \sigma=\frac{e^{2}}{4 \pi^{2}}\left\{\ln \left[\tau_{\uparrow}\left(\frac{1}{\tau_{\varphi \uparrow}}+\frac{1}{\tilde{\tau}_{s o \uparrow}}\right)\right]+\ln \left[\tau_{\downarrow}\left(\frac{1}{\tau_{\varphi \downarrow}}+\frac{1}{\tilde{\tau}_{s o \downarrow}}\right)\right]\right\}
$$


which is a direct generalization of thecorresponding formula in the 2D nonmagnetic case 6.6 Here, $\tau_{\uparrow(\downarrow)}$ and $\tilde{\tau}_{s o \uparrow(\downarrow)}$ are defined, respectively, by (31) and $\tilde{\tau}_{s o \uparrow(\downarrow)}=0$ for the in-plane magnetization, and by Eqs. (34) and (35) for the case of perpendicular magnetization.

The 2D localization correction, described by Eq. (36a), is negative since $\tau<\tilde{\tau}_{s o}, \tau_{\varphi}$, and, in addition, we take here $\tau \ll \tilde{\tau}_{s o}, \tau_{\varphi}$. The latter inequality means that the momentum relaxation time of electrons, $\tau$, is mainly due to the potential scattering.

We can also present an expression for the conductivity in the case of nonzero magnetic induction $\mathbf{B}$, perpendicular to the plane, by generalizing the result for a nonmagnetic two-dimensional system 20

$$
\begin{aligned}
& \Delta \sigma(B)=-\frac{e^{2}}{4 \pi^{2}}\left[\psi\left(\frac{1}{2}+\frac{1}{\tau_{\uparrow} a_{\uparrow}}\right)-\psi\left(\frac{1}{2}+\frac{1}{\tilde{\tau}_{s o \uparrow} a_{\uparrow}}+\frac{1}{\tau_{\varphi \uparrow} a_{\uparrow}}\right)\right. \\
& \left.+\psi\left(\frac{1}{2}+\frac{1}{\tau_{\downarrow} a_{\downarrow}}\right)-\psi\left(\frac{1}{2}+\frac{1}{\tilde{\tau}_{s o \downarrow} a_{\downarrow}}+\frac{1}{\tau_{\varphi \downarrow} a_{\downarrow}}\right)\right], \quad(36 b)
\end{aligned}
$$

where $a_{\uparrow, \downarrow}=4 e B D_{\uparrow, \downarrow} / c$, and $\psi(x)$ is the Digamma function 34 which has the property $\psi(x) \simeq \ln (x)$ for $x \gg 1$.

The magnetic induction suppresses the negative correction to the conductivity, which leads to the negative magnetoresistance. It should be noted that in strongly $2 \mathrm{D}$ case, the in-plane magnetic induction does not affect the localization correction to conductivity. The reason is that in the two-dimensional case, the flux of magnetic induction does not penetrate through any closed electron paths. Correspondingly, the in-plane induction does not break the interference of closed trajectories of electrons moving in opposite firections, responsible for the weak localization effect 6

\section{QUANTUM WELLS}

In a quasi $2 \mathrm{D}$ case the electrons are confined within a quantum well and the number $N_{F}$ of $2 \mathrm{D}$ subbands populated with electrons is larger than one, $N_{F}>1$.
The situation with a large number of occupied subbands is typical for metals, whereas the situation with only a few populated subbands is characteristic of semiconductor quantum wells. The effect of SO scattering on the magnetoresistance of nonmagnetic materials at such conditions has been studied by Hikami et al 20

We consider here a ferromagnetic quantum well in the geometry shown in Fig. 6, i.e., when the magnetization field $\mathbf{M}$ is in the film plane. The scattering amplitude has then the following form

$$
\Gamma_{\alpha \beta \gamma \delta}^{0}=a \delta_{\alpha \delta} \delta_{\beta \gamma}-b \sigma_{\alpha \delta}^{x} \sigma_{\beta \gamma}^{x}-c \sigma_{\alpha \delta}^{y} \sigma_{\beta \gamma}^{y}-t_{\alpha \beta \gamma \delta},
$$

with $a, b$ and $t_{\alpha \beta \gamma \delta}$ defined by Eqs. (4) to (6), and

$$
\begin{gathered}
c=N_{i} V_{1}^{2}{\overline{\left(\mathbf{k} \times \mathbf{k}^{\prime}\right)_{y}^{2}}}^{(\uparrow, \downarrow)}=\frac{1}{2 \pi \nu_{\uparrow} \tau_{\text {so }}^{y}}=\frac{1}{2 \pi \nu_{\downarrow} \tau_{\text {so } \downarrow}^{y}}, \\
d_{1}=N_{i} V_{1}^{2}{\overline{\left(\mathbf{k} \times \mathbf{k}^{\prime}\right)_{z}^{2}}}^{(\uparrow)}=\frac{1}{2 \pi \nu_{\uparrow} \tau_{\text {so }}^{z}}, \\
d_{2}=N_{i} V_{1}^{2}{\overline{\left(\mathbf{k} \times \mathbf{k}^{\prime}\right)_{z}^{2}}}^{(\downarrow)}=\frac{1}{2 \pi \nu_{\downarrow} \tau_{s o \downarrow}^{z}}, \\
d_{3}=-N_{i} V_{1}^{2}{\overline{\left(\mathbf{k} \times \mathbf{k}^{\prime}\right)_{z}^{2}}}^{(\uparrow, \downarrow)} .
\end{gathered}
$$

The averages in Eqs. (38) to (41) include averaging over discrete subbands due to quantization of the electron motion along the axis $x$ (normal to the film plane). In this notation, the expressions for bare vertices $\Gamma_{\uparrow}^{0}$ and $\Gamma_{\downarrow}^{0}$ coincide with Eqs. (11) and (12), whereas for the spin-flip vertex $\Gamma_{s f}^{0}$ we have

$$
\Gamma_{s f}^{0}=-\frac{1}{2 \pi \nu_{\uparrow} \tau_{s o \uparrow}^{x}}+\frac{1}{2 \pi \nu_{\uparrow} \tau_{s o \uparrow}^{y}}
$$

The ladder equations for $\Gamma_{\uparrow}(\omega, q), \quad \Gamma_{\downarrow}(\omega, q)$ and $\Gamma_{s f}(\omega, q)$ have the form shown in Fig. 7. From these equations one finds the following solution for $\Gamma_{\uparrow}(\omega, q)$ :

$$
\Gamma_{\uparrow}(\omega, q)=\frac{\left(\Gamma_{s f}^{0}\right)^{2} \Pi_{\downarrow}(\omega, q)+\Gamma_{\uparrow}^{0}\left[1-\Gamma_{\downarrow}^{0} \Pi_{\downarrow}(\omega, q)\right]}{\left[1-\Gamma_{\uparrow}^{0} \Pi_{\uparrow}(\omega, q)\right]\left[1-\Gamma_{\downarrow}^{0} \Pi_{\downarrow}(\omega, q)\right]-\left(\Gamma_{s f}^{0}\right)^{2} \Pi_{\uparrow}(\omega, q) \Pi_{\downarrow}(\omega, q)} .
$$

In the case of weak SO interaction, the final expression for $\Gamma_{\uparrow}(\omega, q)$ takes the form

$$
\Gamma_{\uparrow}(\omega, q)=\frac{1}{2 \pi \nu \uparrow \tau_{\uparrow}^{2}} \frac{A_{\uparrow}}{-i \omega+\widetilde{D}_{\uparrow} q^{2}+1 / \tilde{\tau}_{s o \uparrow}+1 / \tau_{\varphi \uparrow}},
$$

where

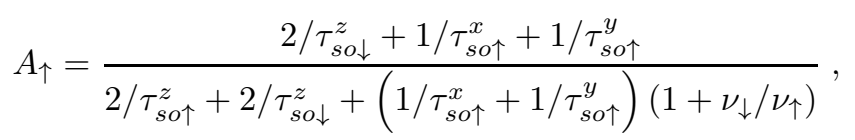




$$
\begin{gathered}
\widetilde{D}_{\uparrow}=\frac{D_{\uparrow}\left(2 / \tau_{s o \downarrow}^{z}+1 / \tau_{s o \uparrow}^{x}+1 / \tau_{s o \uparrow}^{y}\right)+D_{\downarrow}\left(2 / \tau_{s o \uparrow}^{z}+1 / \tau_{s o \downarrow}^{x}+1 / \tau_{s o \downarrow}^{y}\right)}{2 / \tau_{s o \uparrow}^{z}+2 / \tau_{s o \downarrow}^{z}+\left(1 / \tau_{s o \uparrow}^{x}+1 / \tau_{s o \uparrow}^{y}\right)\left(1+\nu_{\downarrow} / \nu_{\uparrow}\right)}, \\
\frac{1}{\tilde{\tau}_{s o \uparrow}}=2 \frac{2 /\left(\tau_{s o \uparrow}^{z} \tau_{s o \downarrow}^{z}\right)+\left(1 / \tau_{s o \uparrow}^{x}+1 / \tau_{s o \uparrow}^{y}\right)\left(1 / \tau_{s o \uparrow}^{z}+\nu_{\downarrow} /\left(\nu_{\uparrow} \tau_{s o \downarrow}^{y}\right)\right)+2 /\left(\tau_{s o \uparrow}^{x} \tau_{s o \downarrow}^{y}\right)}{2 / \tau_{s o \uparrow}^{z}+2 / \tau_{s o \downarrow}^{z}+\left(1 / \tau_{s o \uparrow}^{x}+1 / \tau_{s o \uparrow}^{y}\right)\left(1+\nu_{\downarrow} / \nu_{\uparrow}\right)},
\end{gathered}
$$

and the spin-up relaxation time $\tau_{\uparrow}$ is

$$
\frac{1}{\tau_{\uparrow}}=\frac{1}{\tau_{0 \uparrow}}+\frac{1}{\tau_{s o \uparrow}^{z}}+\frac{1}{\tau_{s o \downarrow}^{x}}+\frac{1}{\tau_{s o \downarrow}^{y}} .
$$

The corresponding expression for $\Gamma_{\downarrow}(\omega, q)$ can be obtained from Eqs. (44) to (48) by changing the arrow direction in these formulas. It is worth to note, that according to Eq. (46), the spin-orbit interaction renormalizes the diffusion coefficients.

The correction to conductivity is described either by Eq. (21) or by Eq. (36), in dependence on the ratio of $L_{e f f}=\left(\widetilde{D} /\left(\tilde{\tau}_{s o}^{-1}+\tau_{\varphi}^{-1}\right)\right)^{1 / 2}$ to the width of the quantum well $L$. For $L_{e f f}<L$ we have effectively a $3 \mathrm{D}$ case described by Eq. (21), whereas for $L_{\text {eff }} \gg L$ one finds effectively $2 \mathrm{D}$ behavior, Eq. (36a). It is worth to note that due to a strong asymmetry between spin-up and spin-down states, a "mixed" case is possible, with a 3D correction for one spin orientation and a 2D correction for the opposite spin orientation.

\section{SPIN-ORBIT INTERACTION OF THE BYCHKOV-RASHBA TYPE}

The case when the spin-orbit coupling enters the Hamiltonian also in the absence of scattering defects needs a special treatment. In the following we will consider a system described by the Haniltonian including the Bychkov-Rashba interaction term 23

$$
H=\int \frac{d^{3} \mathbf{k}}{(2 \pi)^{3}} \psi_{\mathbf{k}}^{\dagger}\left[\frac{k^{2}}{2 m}-M \sigma_{z}+g\left(k_{y} \sigma_{z}-k_{z} \sigma_{y}\right)\right] \psi_{\mathbf{k}},
$$

where the magnetization $\mathbf{M}$ is assumed to be in the plane of the system (the geometry of Fig. 6) and $g \equiv g(k)$ is the spin-orbit coupling parameter. This Hamiltonian can describe electrons in low-dimensional structures close to interfaces, or electrons in a quantum well with variable doping.

The energy spectrum of the Hamiltonian (49) has two branches,

$$
\varepsilon_{1(2)}(\mathbf{k})=\frac{k^{2}}{2 m} \pm\left[\left(M-g k_{y}\right)^{2}+g^{2} k_{z}^{2}\right]^{1 / 2},
$$

which are no longer pure spin-up and spin-down states, but correspond to spin-mixed states. The eigenfunctions corresponding to the eigenvalues (50) can be written as

$$
\begin{gathered}
|1 \mathbf{k}\rangle=\left(4 M^{2}+g^{2} k_{z}^{2}\right)^{-1 / 2}\left(-i g k_{z}|\mathbf{k} \uparrow\rangle+2 M|\mathbf{k} \downarrow\rangle\right) \\
|2 \mathbf{k}\rangle=\left(4 M^{2}+g^{2} k_{z}^{2}\right)^{-1 / 2}\left(2 M|\mathbf{k} \uparrow\rangle+i g k_{z}|\mathbf{k} \downarrow\rangle\right)
\end{gathered}
$$

Due to the terms linear in $k_{y}$ and for $M \neq 0$, the energy spectrum (50) is not symmetrical with respect to the $\mathbf{k} \rightarrow-\mathbf{k}$ transformation, as can also be seen in Fig. 9, (a) and (b).
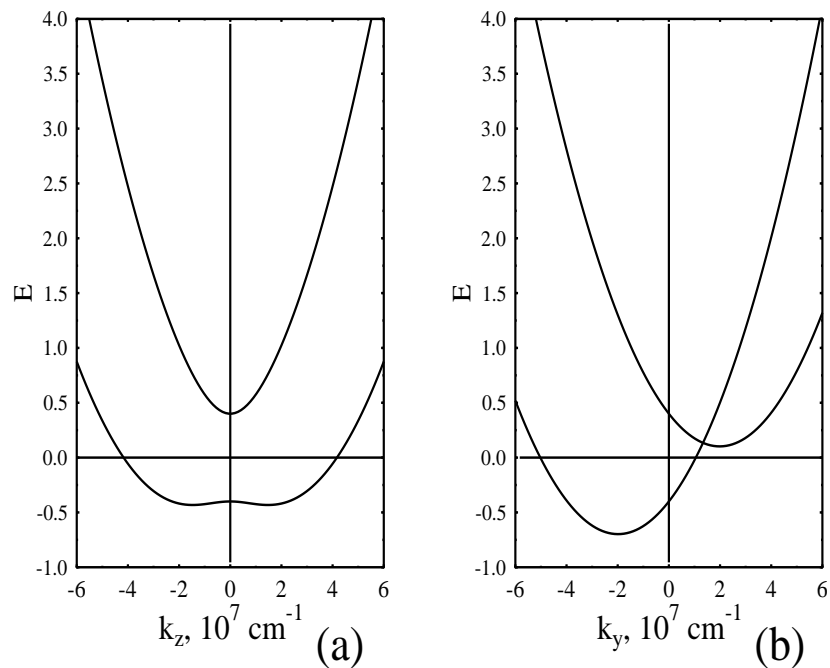

FIG. 9. The energy spectrum $\mathrm{E}(\mathbf{k})$ of a ferromagnet with the Bychkov-Rashba SO coupling, shown as a function of $k_{z}$ at $k_{x}=k_{y}=0$ (a) and as a function of $k_{y}$ at $k_{x}=k_{z}=0(\mathrm{~b})$.

In the absence of external electric field $E$, there are nonvanishing spin currents, associated with each branch of the spectrum $\varepsilon_{1,2}(\mathbf{k})$. However, it can be verified that the total charge current is zero if $E=0$.

In the basis of the spin-up and spin-down states, the electron Green function has the matrix form,

$G_{0}(\varepsilon, \mathbf{k})=\frac{\varepsilon-k^{2} / 2 m+\mu-M \sigma_{z}+g k_{y} \sigma_{z}-g k_{z} \sigma_{y}}{\left(\varepsilon-\varepsilon_{1 \mathbf{k}}+\mu+i \delta \operatorname{sign} \varepsilon\right)\left(\varepsilon-\varepsilon_{2 \mathbf{k}}+\mu+i \delta \operatorname{sign} \varepsilon\right)}$,

where $\mu$ is the chemical potential. Accordingly, the self energy is also a matrix in this basis. It is, however, more 
convenient to consider the electron self energy $\Sigma(\varepsilon, \mathbf{k})$ in the basis of the eigenfunctions (51) and (52). The self energy is then diagonal and the imaginary parts of $\Sigma_{1}\left(0, k_{F 1}\right)$ and $\Sigma_{2}\left(0, k_{F 2}\right)$ give the relaxation times $\tau_{1}$ and $\tau_{2}$ in the presence of defects.

Using Eqs. (51) and (52), one can calculate the matrix elements of the impurity potential $V_{0}(\mathbf{r})$,

$$
V_{1 \mathbf{k}, 1 \mathbf{k}^{\prime}}=V_{2 \mathbf{k}, 2 \mathbf{k}^{\prime}}=\frac{V_{0}\left(4 M^{2}+g g^{\prime} k_{z} k_{z}^{\prime}\right)}{\left(4 M^{2}+g^{2} k_{z}^{2}\right)^{1 / 2}\left(4 M^{2}+g^{\prime 2} k_{z}^{\prime 2}\right)^{1 / 2}}
$$

$V_{1 \mathbf{k}, 2 \mathbf{k}^{\prime}}=-V_{2 \mathbf{k}, 1 \mathbf{k}^{\prime}}=\frac{2 i V_{0} M\left(g k_{z}+g^{\prime} k_{z}^{\prime}\right)}{\left(4 M^{2}+g^{2} k_{z}^{2}\right)^{1 / 2}\left(4 M^{2}+g^{\prime 2} k_{z}^{\prime 2}\right)^{1 / 2}}$,

where $g^{\prime}=g\left(k^{\prime}\right)$.

To find the self energy one needs to calculate the diagrams shown in Fig. 10.
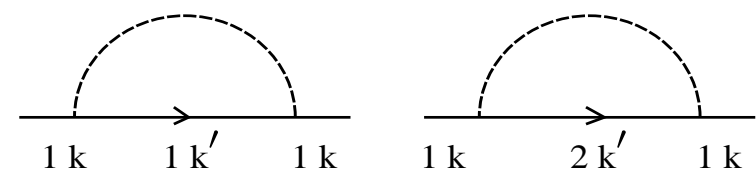

FIG. 10. Self energy in the case of the Bychkov-Rashba interaction.

In what follows we consider the case when SO is small as compared to the magnetic splitting, so we can expand the self energy in a small parameter $g k_{F} / M \ll 1$. Then, we find

$\Sigma_{1}^{R}(\varepsilon, \mathbf{k}) \simeq N_{i} V_{0}^{2}\left(1+\frac{g^{2} k_{z}^{2}}{4 M^{2}}\right) \int \frac{d^{3} \mathbf{k}^{\prime}}{(2 \pi)^{3}}\left(1-\frac{g^{\prime 2} k_{z}^{\prime 2}}{4 M^{2}}\right) \frac{1}{\varepsilon-\varepsilon_{1}\left(\mathbf{k}^{\prime}\right)+\mu+i \delta}+N_{i} V_{0}^{2} \int \frac{d^{3} \mathbf{k}^{\prime}}{(2 \pi)^{3}} \frac{g^{\prime 2} k_{z}^{\prime 2}}{4 M^{2}} \frac{1}{\varepsilon-\varepsilon_{2}\left(\mathbf{k}^{\prime}\right)+\mu+i \delta}$

and a similar expression for $\Sigma_{2}^{R}(\varepsilon, \mathbf{k})$. In these formulas we can put $\varepsilon=0, k=k_{F \downarrow(\uparrow)}$, and average over the Fermi surfaces. This leads to the relaxation times for electrons corresponding to the bands 1 and 2,

$$
\begin{gathered}
\tau_{1}=\frac{1}{2 \pi \nu_{1} N_{i} V_{0}^{2}}\left(1-\frac{\nu_{\uparrow}}{\nu_{\downarrow}} \frac{g_{\uparrow}^{2} k_{F \uparrow}^{2}}{12 M^{2}}\right), \\
\tau_{2}=\frac{1}{2 \pi \nu_{2} N_{i} V_{0}^{2}}\left(1-\frac{\nu_{\downarrow}}{\nu_{\uparrow}} \frac{g_{\downarrow}^{2} k_{F \downarrow}^{2}}{12 M^{2}}\right),
\end{gathered}
$$

where $g_{\uparrow}=g\left(k_{F \uparrow}\right)$ and $g_{\downarrow}=g\left(k_{F \downarrow}\right)$. The above formulae explicitly show the contribution of SO interaction to the relaxation time.

We also need to calculate the densities of states $\nu_{1}$ and $\nu_{2}$ at the Fermi surfaces and to relate them to the ones in the absence of the spin-orbit interaction, $\nu_{\downarrow}$ and $\nu_{\uparrow}$. To do this we write

$$
\nu_{1(2)}=\int \frac{d^{3} \mathbf{k}}{(2 \pi)^{3}} \delta\left(\mu-\varepsilon_{1(2)}(\mathbf{k})\right),
$$

and then, upon integrating over $k_{x}$, we obtain

$$
\nu_{1(2)}=\frac{(2 m)^{1 / 2}}{(2 \pi)^{3}} \int_{\varepsilon_{1(2)}^{l}(\mathbf{k})<\mu} \frac{d k_{y} d k_{z}}{\left(\mu-\varepsilon_{1(2)}^{l}(\mathbf{k})\right)^{1 / 2}}
$$

where

$$
\varepsilon_{1(2)}^{l}(\mathbf{k})=\frac{k_{y}^{2}+k_{z}^{2}}{2 m} \pm s_{\mathbf{k}}
$$

$$
s_{\mathbf{k}}=\left[\left(M-g k_{y}\right)^{2}+g^{2} k_{z}^{2}\right]^{1 / 2}
$$

Expanding in powers of $g k_{F} / M \ll 1$ and calculating the integrals in (60), we find

$$
\nu_{1}=\nu_{\downarrow}\left(1+\frac{m^{2} g_{\downarrow}^{2}}{2 k_{F \downarrow}^{2}}-\frac{m g_{\downarrow}^{2}}{2 M}\right)
$$

and

$$
\nu_{2}=\nu_{\uparrow}\left(1+\frac{m^{2} g_{\uparrow}^{2}}{2 k_{F \uparrow}^{2}}-\frac{m g_{\uparrow}^{2}}{2 M}\right)
$$

The relaxation times $\tau_{1}$ and $\tau_{2}$ enter the expressions for the renormalized retarded $\left(G^{R}\right)$ and advanced $\left(G^{A}\right)$ Green functions

$G^{R, A}(\varepsilon, \mathbf{k})=\frac{\varepsilon-k^{2} / 2 m+\mu-M \sigma_{z}+g k_{y} \sigma_{z}-g k_{z} \sigma_{y}}{\left(\varepsilon-\varepsilon_{1 \mathbf{k}}+\mu \pm i / 2 \tau_{1}\right)\left(\varepsilon-\varepsilon_{2 \mathbf{k}}+\mu \pm i / 2 \tau_{2}\right)}$.

Now we have already all quantities necessary to calculate the renormalized vertex $\Gamma_{\alpha \beta \gamma \delta}$. The general ladder-type equation for such a vertex reads

$$
\Gamma_{\alpha \beta \gamma \delta}(\omega, q)=N_{i} V_{0}^{2} \delta_{\alpha \delta} \delta_{\beta \gamma}
$$

$$
+N_{i} V_{0}^{2} \sum_{\nu s} \Pi_{\alpha \nu \beta s}(\omega, q) \Gamma_{\nu s \gamma \delta}(\omega, q)
$$


where $V_{0}$ is the matrix element of the short-range impurity potential, and

$$
\Pi_{\alpha \nu \beta s}(\omega, q)=\int \frac{d^{3} \mathbf{k}}{(2 \pi)^{3}} G_{\alpha \nu}^{R}(\omega, \mathbf{k}+\mathbf{q}) G_{\beta s}^{A}(0,-\mathbf{k}) .
$$

As before, we will restrict ourselves by considering the coupling constant $g$ to be small as compared to the spin splitting, $g k_{F} / M \ll 1$.

Using Eqs. (66) and (67), and integrating over $k_{x}$, we find for $q, \omega=0$

$$
\begin{aligned}
\Pi_{\alpha \nu \beta s}(0,0) & =\frac{i(2 m)^{1 / 2}}{8 \pi^{2}} \times\left[\int_{\varepsilon_{1}^{l}(\mathbf{k})<\mu} d k_{y} d k_{z} \frac{\left[s_{\mathbf{k}}-M \sigma_{z}+g\left(k_{y} \sigma_{z}-k_{z} \sigma_{y}\right)\right]_{\alpha \nu}\left[s_{\mathbf{k}}-M \sigma_{z}-g\left(k_{y} \sigma_{z}-k_{z} \sigma_{y}\right)\right]_{\beta s}}{s_{\mathbf{k}}\left(s_{\mathbf{k}}+s_{-\mathbf{k}}\right)\left(s_{-\mathbf{k}}-s_{\mathbf{k}}+i / \tau_{1}\right)\left[\mu-\left(k_{y}^{2}+k_{z}^{2}\right) / 2 m-s_{\mathbf{k}}\right]^{1 / 2}}\right. \\
& \left.+\int_{\varepsilon_{2}^{l}(\mathbf{k})<\mu} d k_{y} d k_{z} \frac{\left[-s_{\mathbf{k}}-M \sigma_{z}+g\left(k_{y} \sigma_{z}-k_{z} \sigma_{y}\right)\right]_{\alpha \nu}\left[-s_{\mathbf{k}}-M \sigma_{z}-g\left(k_{y} \sigma_{z}-k_{z} \sigma_{y}\right)\right]_{\beta s}}{s_{\mathbf{k}}\left(s_{\mathbf{k}}+s_{-\mathbf{k}}\right)\left(s_{\mathbf{k}}-s_{-\mathbf{k}}+i / \tau_{2}\right)\left[\mu-\left(k_{y}^{2}+k_{z}^{2}\right) / 2 m+s_{\mathbf{k}}\right]^{1 / 2}}\right] .
\end{aligned}
$$

If we put $g=0$ in Eq. (68), we find that only two matrix elements are nonzero, namely $\Pi_{\uparrow \uparrow \uparrow \uparrow}(0,0)$ and $\Pi_{\downarrow \downarrow \downarrow \downarrow}(0,0)$. Assuming $D q^{2} \tau, \omega \tau \ll 1$, we can just supplement this result by the terms of the expansion in small $q$ and $\omega$

$$
\Pi_{\uparrow \uparrow \uparrow \uparrow}(\omega, q)=2 \pi \nu_{\uparrow} \tau_{\uparrow}\left(1-D_{\uparrow} q^{2} \tau_{\uparrow}+i \omega \tau_{\uparrow}\right),
$$

and a similar one for $\Pi_{\downarrow \downarrow \downarrow \downarrow}(\omega, q)$.

Let us consider now the limit of weak SO coupling and expand $\Pi_{\alpha \nu \beta s}(q, \omega)$ in powers of $g k_{F} / M$. The first non-vanishing term of this expansion is quadratic in this parameter. According to Eq. (68), the other small parameter in the limit of $g \rightarrow 0$ is $g k_{F} \tau$. Thus, if we assume $M \tau \ll 1$, we can neglect the terms $\sim\left(g k_{F} \tau\right)^{2}$ and keep the terms $\sim g k_{F} / M$. In the opposite limit, $M \tau \gg 1$ (clean ferromagnet), we should keep terms $\sim\left(g k_{F} \tau\right)^{2}$ and neglect terms $\sim g k_{F} / M$. Since our considerations of the localization corrections are limited by $\varepsilon_{F} \tau \gg 1$, the dirty case is possible only for some weak ferromagnets, when $M \ll \varepsilon_{F}$ (in other words, for ferromagnets with very low polarization).
In view of Eq.(49), $g k_{F}$ is the amplitude of the spinflip process. In the classical picture $g k_{F}$ is the angle of spin rotation in the unit time. Hence, $g k_{F} \tau$ is just the angle of the classical spin rotation due to the BychkovRashba perturbation at the mean free path $l$ of the electron. Thus, the smallness of the parameter $g k_{F} \tau$ corresponds to a small spin rotation angle at the length $l$.

Since considerations in this paper are restricted to the case $M \tau \gg 1$, we will not discuss here the opposite limit, $M \tau \ll 1$. Such a case needs a special treatment. Indeed, as we pointed out in Sec. 1, only the condition $M \tau \gg 1$ allows to restrict oneselves to the triplet Cooper channel. The result on the only nonvanishing matrix elements $\Pi_{\uparrow \uparrow \uparrow \uparrow}(0,0)$ and $\Pi_{\downarrow \downarrow \downarrow \downarrow \downarrow}(0,0)$ for $g=0$ (see Eqs. (68) and (69)) also refers to the case $M \tau \gg 1$ since in Eqs. (65) and (68) we account for the self energy as a small shift of poles of the Green function from the real axis.

In the limit of small SO interaction, $g k_{F} \tau \ll 1$, and for $M \tau \gg 1$, we can neglect in Eq. (68) all terms of the order of $g k_{F} / M$. As a result, we find only two nonvanishing matrix elements

$$
\begin{aligned}
& \Pi_{\uparrow \uparrow \uparrow \uparrow}(\omega, q) \equiv \Pi_{\uparrow}(\omega, q)=2 \pi \nu_{\uparrow} \tau_{\uparrow}\left(1-D_{\uparrow} q^{2} \tau_{\uparrow}+i \omega \tau_{\uparrow}-\frac{4}{3} g_{\uparrow}^{2} \tau_{\uparrow}^{2} k_{F \uparrow}^{2}\right), \\
& \Pi_{\downarrow \downarrow \downarrow \downarrow}(\omega, q) \equiv \Pi_{\downarrow}(\omega, q)=2 \pi \nu_{\downarrow} \tau_{\downarrow}\left(1-D_{\downarrow} q^{2} \tau_{\downarrow}+i \omega \tau_{\downarrow}-\frac{4}{3} g_{\downarrow}^{2} \tau_{\downarrow}^{2} k_{F \downarrow}^{2}\right),
\end{aligned}
$$

which give rise to the Cooperon $\Gamma_{\uparrow}(\omega, q)$ in the form of Eq. (18), with the effective spin-orbit relaxation time

$$
\frac{1}{\tilde{\tau}_{s o \uparrow}}=\frac{4}{3} g_{\uparrow}^{2} k_{F \uparrow}^{2} \tau_{\uparrow} .
$$

Similar expressions can be obtained for $\Gamma_{\downarrow}(\omega, q)$ and $\tilde{\tau}_{s o \downarrow}$.

If we take the limit $g k_{F} \tau \gg 1$, then for $M \tau \gg 1$ we still can consider the SO interaction as a small perturbation, $g k_{F} / M \ll 1$. Calculating the integral (68) up to the second order in this parameter, we find

$$
\Pi_{\alpha \nu \beta s}(\omega, q)=\frac{3 \pi \nu_{\downarrow} \tau_{1}}{8\left(g_{\downarrow} k_{F \downarrow} \tau_{1}\right)^{2}}\left[\left(1-D_{\downarrow} q^{2} \tau_{1}+i \omega \tau_{1}-\frac{m g_{\downarrow}^{2}}{2 M}-\frac{g_{\downarrow}^{2} k_{F \downarrow}^{2}}{3 M^{2}}+\frac{m^{2} g_{\downarrow}^{2}}{2 k_{F \downarrow}^{2}}\right)\left(1-\sigma_{z}\right)_{\alpha \nu}\left(1-\sigma_{z}\right)_{\beta s}\right.
$$




$$
\begin{gathered}
\left.-\frac{g_{\downarrow}^{2} k_{F \downarrow}^{2}}{3 M^{2}} \sigma_{\alpha \nu}^{y} \sigma_{\beta s}^{y}+\frac{g_{\downarrow}^{2} k_{F \downarrow}^{2}}{6 M^{2}} \delta_{\alpha \nu}\left(1-\sigma_{z}\right)_{\beta s}+\frac{g_{\downarrow}^{2} k_{F \downarrow}^{2}}{6 M^{2}}\left(1-\sigma_{z}\right)_{\alpha \nu} \delta_{\beta s}-\frac{m g_{\downarrow}^{2}}{M}\left(1-\sigma_{z}\right)_{\alpha \nu}\left(1+\sigma_{z}\right)_{\beta s}\right] \\
\quad+\frac{3 \pi \nu_{\uparrow} \tau_{2}}{8\left(g_{\uparrow} k_{F \uparrow} \tau_{2}\right)^{2}}\left[\left(1-D_{\uparrow} q^{2} \tau_{2}+i \omega \tau_{2}+\frac{m g_{\uparrow}^{2}}{2 M}-\frac{g_{\uparrow}^{2} k_{F \uparrow}^{2}}{3 M^{2}}+\frac{m^{2} g_{\uparrow}^{2}}{2 k_{F \uparrow}^{2}}\right)\left(1+\sigma_{z}\right)_{\alpha \nu}\left(1+\sigma_{z}\right)_{\beta s}\right. \\
\left.-\frac{g_{\uparrow}^{2} k_{F \uparrow}^{2}}{3 M^{2}} \sigma_{\alpha \nu}^{y} \sigma_{\beta s}^{y}+\frac{g_{\uparrow}^{2} k_{F \uparrow}^{2}}{6 M^{2}} \delta_{\alpha \nu}\left(1+\sigma_{z}\right)_{\beta s}+\frac{g_{\uparrow}^{2} k_{F \uparrow}^{2}}{6 M^{2}}\left(1+\sigma_{z}\right)_{\alpha \nu} \delta_{\beta s}+\frac{m g_{\uparrow}^{2}}{M}\left(1+\sigma_{z}\right)_{\alpha \nu}\left(1-\sigma_{z}\right)_{\beta s}\right] .
\end{gathered}
$$

Using (73), we can find all nonvanishing matrix elements of $\Pi_{\alpha \nu \beta s}(\omega, q)$ and then solve the ladder equations of Fig. 7. We do not make this calculation since we notice that the presence of the small factor $1 /\left(g k_{F} \tau\right)^{2}$ leads to complete suppresion of the Cooperon. Thus, the localization corrections exist only for $g k_{F} \tau<1$.

Finally, we present the calculation of a classical correction to the conductivity due to the SO interaction in Hamiltomian (49). After calculating the loop diagram with the Green functions (65), and using (57),(58),(63),(64), we find

$$
\sigma_{z z}^{(0)}=\frac{e^{2} n_{\uparrow} \tau_{\uparrow}}{m}\left(1+\frac{m^{2} g_{\uparrow}^{2}}{k_{F \uparrow}^{2}}-\frac{m g_{\uparrow}^{2}}{M}-\frac{\nu_{\downarrow}}{\nu_{\uparrow}} \frac{g_{\downarrow}^{2}}{12 M^{2}}\right)+\frac{k^{2} n_{\downarrow} \tau_{\downarrow}}{m}\left(1+\frac{m^{2} g_{\downarrow}^{2}}{k_{F \downarrow}^{2}}-\frac{m g_{\downarrow}^{2}}{M}-\frac{\nu_{\uparrow}}{\nu_{\downarrow}} \frac{g_{\uparrow}^{2} k_{F \uparrow}^{2}}{12 M^{2}}\right) .
$$

This expression contains corrections related to renormalization of the density of states (second and third terms in each bracket) and of the scattering time (fourth terms).

We should also take into account a renormalization of the chemical potential $\mu$. For this purpose we calculate the total number of spin up and down electrons and impose the condition of constant particle number. After simple calculations we find the correction to the chemical potential

$$
\Delta \mu=\frac{g_{\uparrow}^{2} k_{F \uparrow}^{3}+g_{\downarrow}^{2} k_{F \downarrow}^{3}}{6 M\left(k_{F \uparrow}+k_{F \downarrow}\right)}-\frac{m\left(g_{\uparrow}^{2} k_{F \uparrow}+g_{\downarrow}^{2} k_{F \downarrow}\right)}{2\left(k_{F \uparrow}+k_{F \downarrow}\right)} .
$$

Calculating the corresponding corrections to Fermi momenta, $k_{F \uparrow, \downarrow}$, we find the corrections to spin up and down classical conductivities connected with renormalization of the chemical potential

$$
\begin{aligned}
& \frac{\Delta \sigma_{z z \uparrow}^{(0)}}{\sigma_{z z}^{(0)}}=\frac{m\left(g_{\uparrow}^{2} k_{F \uparrow}^{3}+g_{\downarrow}^{2} k_{F \downarrow}^{3}\right)}{2 M k_{F \uparrow}^{2}\left(k_{F \uparrow}+k_{F \downarrow}\right)}-\frac{3 m^{2}\left(g_{\uparrow}^{2} k_{F \uparrow}+g_{\downarrow}^{2} k_{F \downarrow}\right)}{2 k_{F \uparrow}^{2}\left(k_{F \uparrow}+k_{F \downarrow}\right)}, \\
& \frac{\Delta \sigma_{z z \downarrow}^{(0)}}{\sigma_{z z}^{(0)}}=\frac{m\left(g_{\uparrow}^{2} k_{F \uparrow}^{3}+g_{\downarrow}^{2} k_{F \downarrow}^{3}\right)}{2 M k_{F \downarrow}^{2}\left(k_{F \uparrow}+k_{F \downarrow}\right)}-\frac{3 m^{2}\left(g_{\uparrow}^{2} k_{F \uparrow}+g_{\downarrow}^{2} k_{F \downarrow}\right)}{2 k_{F \downarrow}^{2}\left(k_{F \uparrow}+k_{F \downarrow}\right)} .
\end{aligned}
$$

It should be noted that the classical corrections, (76) and (77), can be of any sign since the inequality $M \gg 1 / \tau$ does not necessarily imply any relations between the magnitude of $M$ and the Fermi energies of majority and minority electrons.

The total correction to conductivity includes all corrections to the classical part, Eqs. (74), (76) and (77), as well as the quantum correction in the form of Eqs. (21) or $(36 \mathrm{a}, \mathrm{b})$ with $\tilde{\tau}_{s o}$, given by $(72)$ for $g k_{F} \tau<1$.

\section{EFFECT OF INTERNAL MAGNETIC INDUCTION IN A FERROMAGNET}

In our model we have taken into account the effect of magnetic induction $\mathbf{B}$, which enters the kinetic energy of electrons through a vector potential $\mathbf{A}$ and leads to the suppression of localization corrections, Eqs. (22) and (36b). The total magnetic induction $\mathbf{B}$ inside a ferromagnet includes the external magnetic field $\mathbf{H}^{\text {ext }}$ and the internal magnetic induction $\mathbf{B}^{\text {int }}, \mathbf{B}=\mathbf{H}^{\text {ext }}+\mathbf{B}^{\text {int }}$, where $B_{i}^{i n t}=4 \pi\left(\delta_{i j}-n_{i j}\right) M_{0 j}, \mathbf{M}_{0}$ is the magnetization vector and $n_{i j}$ is the demagnetizing factor tensor. 35 The magnitude of the internal magnetic induction may be rather high in strong ferromagnets. Nevertheless, weak localization corrections were observed, e.g., in Ni films. $\frac{15}{5}$

We can present some numerical estimations of the magnitude of $B^{\text {int }}$. For example, in the case of Fe we take 39 $4 \pi M_{0} \simeq 2 \mathrm{~T}$. Thus, for the bulk Fe, when the demagnetizing field is negligibly small, we have $B^{\text {int }} \simeq 2 \mathrm{~T}$. The critical magnetic induction $B^{\text {crit }}$, which can totally suppress the localizations correction, is determined differently in 3 and $2 \mathrm{D}$ cases. In the $3 \mathrm{D}$ case we can estimated it by $6.8 l_{B} \simeq l$ ( $l$ is the electron mean free path). For the parameters of very pure bulk Fe $\left(m \simeq 4 m_{0}\right.$ and $\tau \simeq 5 \times 10^{-13} \mathrm{~s}$ ), 32 we find $l \simeq 4 \times 10^{-5} \mathrm{~cm}$, and consequently $B_{\text {crit }} \simeq 50$ Oe. This estimation shows that the localization corrections in pure bulk Fe are totally suppressed by the internal magnetic induction. On the other hand, in not so pure metals, or in magnetic alloys, and amorphous materials, one can expect a much 
shorter mean free path, like $l \simeq 10^{-6} \mathrm{~cm}$, which is still large enough for the localization corrections to be small $\left(k_{F} l \gg 1\right)$. In case of $l=10^{-6} \mathrm{~cm}$, one finds $B_{\text {crit }} \simeq 7 \mathrm{~T}$, which is significantly larger than the internal induction of $2 \mathrm{~T}$ and, therefore, makes the localization corrections observable.

In the case of thin magnetic films, the demagnetizing factor is of crucial importance. For example, when the magnetization vector $\mathbf{M}_{0}$ is perpendicular to the interface, the demagnetizing factor is unity, $n_{z z}=1$ ( $z$ axis is perpedicular to the plane; other components of demagnetizing tensor are very small), and we have $\mathbf{B}^{\text {int }}=0$. In this case, the results of Sec. 4 can be applied with the magnetic induction $B$ equal to the external magnetic field $H^{\text {ext }}$. The critical magnetic field, oriented perpendicular to the plane, which suppresses the localization correction, can be estimated as in the 3D case.
On the other hand, in the case of in-plane magnetization, the demagnetizing factor is much smaller than unity, and the non-vanishing internal magnetic induction $\mathbf{B}^{\text {int }}=4 \pi \mathbf{M}_{0}$ is directed along the film. However, the effect of parallel magnetic induction on the localization corrections in a strongly two-dimensional system is absent (Sec. 4). For a quasi-2D system like a quantum well, the effect of parallel induction is non-vanishing but weak. We can write the corresponding expressions for localization corrections in the presence of in-plane magnetic induction by simply generalizing the results of Refs. [ 36, 37.

If the film thickness $L$ is large with respect to the electron mean free path $l$, but stillsmall enough to consider the film as a $2 \mathrm{D}$ system 6 , $l \ll L \ll$ $\left[\left(D \tilde{\tau}_{s o}\right)^{-1}+\left(D \tau_{\varphi}\right)^{-1}\right]^{-1 / 2}$, we can obtain, using Ref. [ 36], the dependence of localization correction on magnetic induction in the limit of small $B$, i.e., for $l_{B} \gg L$ :

$$
\Delta \sigma(B)-\Delta \sigma(0)=\frac{e^{2}}{4 \pi^{2}}\left[\ln \left(1+\frac{L^{2} D_{\uparrow}\left(\tilde{\tau}_{\text {so }}^{-1}+\tau_{\varphi \uparrow}^{-1}\right)^{-1}}{12 l_{B}^{4}}\right)+\ln \left(1+\frac{L^{2} D_{\downarrow}\left(\tilde{\tau}_{s o \downarrow}^{-1}+\tau_{\varphi \downarrow}^{-1}\right)^{-1}}{12 l_{B}^{4}}\right)\right] .
$$

It shows a weaker dependence on the magnetic induction, as compare to the $3 \mathrm{D}$ case. For larger values of $B$, when $l_{B}<L$, the dependence on magnetic induction is as for the 3D case, Eq. (22). The critical value $B_{\text {crit }}$ can also be estimated as for the 3D case. Thus, for thick clean magnetic films, such that $L \gg l$, and $l>l_{0} \equiv\left[c /\left(4 e \pi M_{0}\right)\right]^{1 / 2}$, the in-plane internal magnetic induction suppresses the localization corrections completely. But in dirty or amorphous thick films with $l<l_{0}$, they can be observed. Using the parameters of Fe, we find $l_{0} \simeq 1.8 \times 10^{-6} \mathrm{~cm}$.

If the film thickness $L$ is smaller than the mean free path $l$ (ballistic regime), we use the result of Ref. [ [37], which can be presented in a simple form for some intervals of $B$

To avoid combersome formulae, we introduce the following notations

$$
\frac{1}{\tau_{c \uparrow, \downarrow}}=\frac{1}{\tilde{\tau}_{s o \uparrow, \downarrow}}+\frac{1}{\tau_{\varphi \uparrow, \downarrow}}, \quad L_{c \uparrow, \downarrow}=\left(D_{\uparrow, \downarrow} \tau_{c \uparrow, \downarrow}\right)^{1 / 2} .
$$

When $L_{c}, l_{B}^{2} / L \gg l$, we obtain

$$
\Delta \sigma(B)-\Delta \sigma(0)=\frac{e^{2}}{4 \pi^{2}}\left[\ln \left(1+\frac{l_{\uparrow} L^{3}}{16 l_{B}^{4}} \frac{\tau_{c \uparrow}}{\tau_{\uparrow}}\right)+\ln \left(1+\frac{l_{\downarrow} L^{3}}{16 l_{B}^{4}} \frac{\tau_{c \downarrow}}{\tau_{\downarrow}}\right)\right],
$$

When $L_{c}, l \gg l_{B}^{2} / L$,

$$
\Delta \sigma(B)-\Delta \sigma(0)=\frac{e^{2}}{4 \pi^{2}}\left[\ln \left(1+\frac{L^{2}}{3 l_{B}^{2}} \frac{\tau_{c \uparrow}}{\tau_{\uparrow}}\right)+\ln \left(1+\frac{L^{2}}{3 l_{B}^{2}} \frac{\tau_{c \downarrow}}{\tau_{\downarrow}}\right)\right],
$$

and when $l, l_{B}^{2} / L \gg L_{c}$,

$$
\Delta \sigma(B)-\Delta \sigma(0)=\frac{e^{2}}{4 \pi^{2}} \frac{\pi}{48}\left(\frac{L_{c \uparrow} L^{3}}{l_{B}^{4}} \frac{\tau_{c \uparrow}}{\tau_{\uparrow}}+\frac{L_{c \downarrow} L^{3}}{l_{B}^{4}} \frac{\tau_{c \downarrow}}{\tau_{\downarrow}}\right) .
$$

We also find that the critical in-plane magnetic induction in the case of $L \ll l$ can be estimated from $l_{B} \simeq L$, which gives rise to a much larger critical value of magnetic induction, $B_{\text {thinfilm }}^{\text {crit }} / B_{3 D}^{\text {crit }} \simeq(l / L)^{2} \gg 1$. In other words, even for clean magnetic films, the in-plane magnetic induction does not suppress completely the localization corrections, if the film width is sufficiently small.

In view of the above considerations, the best configuration for observing weak localization effects in a ferromagnet is to use a thin film with perpendicular easy axis, and apply a perpendicular magnetic field. 


\section{SUMMARY AND CONCLUSIONS}

We have analyzed the localization corrections to electrical conductivity in magnetically polarized materials with spin-orbit interactions. The strong magnetic polarization excludes processes with the singlet Cooperon, which are responsible for the antilocalization in nonmagnetic materials with SO scattering. As a result, the quantum correction to conductivity is always negative in ferromagnets and leads to negative magnetoresistance.

The strength of SO interaction, together with the phase relaxation time due to inelastic processes, determine the magnitude of these corrections. In the case when the SO interaction is associated with scattering from impurities and/or other defects, the effective SO scattering time $\tilde{\tau}_{s o}$ entering the Cooperon, depends on the dimensionality of the system and on the magnetization orientation with respect to the plane of the system (in the case of two-dimensional or quasitwo-dimensional systems). In the case of strongly twodimensional ferromagnets with in-plane magnetization, the inverse time $1 / \tilde{\tau}_{s o}$ is zero. This increases the magnitude of the localization correction. The vanishing value of of $1 / \tilde{\tau}_{s o}$ is essentially related to the spin-flip scattering of the Cooperon; the usual contribution from the spinconserving scattering is cancelled by the spin-flip contribution, which enters the Cooperon with an opposite sign. In the quasi-two-dimensional case both contributions are present, but they do not cancel each other.

We have also found the effective spin relaxation time in the case of Bychkov-Rashba SO interaction. It contains contributions from both spin-flip and spin-conserving scattering processes.

We think that good candidates for observations of the localization corrections are also semiconducting ferromagnets like GaMnAs 18 which are recently extensively studied in view of their possible applications in spintronics. Another example is a new ferromagnetic semiconductor $\mathrm{CaB}_{6}$, where a very small magnetization can be combined with a small electron density 40

\section{ACKNOWLEDGEMENTS}

One of authors (V.D.) is thankful to J. Berakdar, A. Crépieux and E. Ya. Sherman for stimulating discussions and valuable comments. V.D. and J.B. thank the Polish Committee for Scientific Research for a support under Grant No. 5 P03B 091 20, and also for a support by NATO Linkage Grant No. 977615.

$†$ Email address: vdugaev@mpi-halle.de
${ }^{1}$ M. N. Baibich, J. M. Broto, A. Fert, F. Nguyen van Dau, F. Petroff, P. Etienne, G. Creuzet, A. Friederich, and J. Chazelas, Phys. Rev. Lett. 61, 2472 (1988).

${ }^{2}$ G. Binasch, P. Grünberg, F. Saurenbach, and W. Zinn, Phys. Rev. B 39, 4828 (1989).

${ }^{3}$ G. A. Prinz, Science 282, 1660 (1998).

${ }^{4}$ R. Fiederling, M. Kelm, G. Reuscher, W. Ossau, G. Schmidt, A. Waag, and L. W. Molenkamp, Nature 402, 787 (1999); Y. Ohno, D. K. Young, B. Beschoten, F. Matsukura, H. Ohno, and D. D. Awschalom, Nature 402, 790 (1999); K. Manyala, Y. Sidis, J. F. DiTusa, G. Aeppli, D. P. Young, and Z. Fisk, Nature 404, 581 (2000).

${ }^{5}$ D. P. DiVincenzo and D. Loss, J. Magn. Magn. Materials 200, 202 (1999); D. P. DiVincenzo, G. Burkard, D. Loss, and E. V. Sukhorukov, cond-mat/9911245.

${ }^{6}$ B. L. Altshuler, A. G. Aronov, D. E. Khmelnitskii, and A. I. Larkin, in Quantum Theory of Solids, edited by I. M. Lifshits (Mir, Moscow, 1982), p. 130-237.

${ }^{7}$ P. A. Lee and T. V. Ramakrishnan, Rev. Mod. Phys. 57, 287 (1985).

${ }^{8}$ B. L. Altshuler and A. G. Aronov, Electron-Electron Interaction in Disordered Systems, edited by A. L. Efros and M. Pollak (Elsevier, Amsterdam, 1985).

${ }^{9}$ G. Bergmann, Phys. Rep. 107, 1 (1984).

${ }^{10}$ A. Singh and E. Fradkin, Phys. Rev. B 35, 6894 (1987).

${ }^{11}$ V. K. Dugaev and J. Barnaś, Europhys. Lett. 54, 105 (2001).

12 S. Kobayashi, Y. Ootuka, F. Komori, and W. Sasaki. J. Phys. Soc. Jpn. 51689 (1982).

${ }^{13}$ H. Raffy, L. Dumoulin, and J. P. Burger, Phys. Rev. B 36, 2158 (1987).

${ }^{14}$ M. Rubinstein, F. J. Rachford, W. W. Fuller, and G. A. Prinz, Phys. Rev. B 37, 8689 (1988).

${ }^{15}$ M. Aprili, J. Lesueur, L. Dumoulin, and P. Nédellec, Solid State Commun. 102, 41 (1997).

${ }^{16}$ F. G. Aliev, E. Kunnen, K. Temst, K. Mae, G. Verbanck, J. Barnaś, V. V. Moshchalkov, and Y. Bruynseraede, Phys. Rev. Lett. 78, 134 (1997).

${ }^{17}$ I. P. Smorchkowa, N. Samarth, J. M. Kikkawa, and D. D. Awschalom, Phys. Rev. Lett. 78, 3571 (1997).

${ }^{18}$ H. Ohno, A. Shen, F. Matsukura, A. Oiwa, A. Endo, S. Katsumoto, and Y. Iye, Appl. Phys. Lett. 69, 363 (1996); H. Ohno, Science 281, 951 (1998); H. Ohno and F. Matsukura, Sol. State Commun. 117, 179 (2001).

${ }^{19}$ P. W. Anderson, E. Abrahams, and T. V. Ramakrishnan, Phys. Rev. Lett. 43, 718 (1979).

${ }^{20}$ S. Hikami, A. I. Larkin, and Y. Nagaoka, Prog. Theor. Phys. 63, 707 (1980).

${ }^{21}$ S. Maekawa and H. Fukuyama, J. Phys. Soc. Jpn. 50, 2516 (1981).

22 G. Dresselhaus, Phys. Rev. 100, 580 (1955).

${ }^{23}$ Yu. A. Bychkov and E. I. Rashba, Pis'ma Zh. Eksp. Teor. Fiz. 39, 64 (1984) [JETP Lett. 39, 78 (1984)]; Yu. A. Bychkov and E. I. Rashba, J. Phys. C 17, 6093 (1984).

${ }^{24}$ A. G. Aronov and Y. B. Lyanda-Geller, Phys. Rev. Lett. 70, 343, 1993.

${ }^{25}$ W. Knap, C. Skierbiszewski, A. Zduniak, E. LitwinStaszewska, D. Bertho, F. Kobbi, J. L. Robert, G. E. Pikus, F. G. Pikus, S. V. Iordanskii, V. Mosser, K. Zekentes, and Yu. B. Lyanda-Geller. Phys. Rev. B 53, 3912 (1996). 
${ }^{26}$ Yu. Lyanda-Geller, Phys. Rev. Lett. 80, 4273 (1998).

27 J. E. Hirsch, Phys. Rev. Lett. 83, 1834 (1999).

${ }^{28}$ E. N. Bulgakov, K. N. Pichugin, A. F. Sadreev, P. Středa, and P. Š eba, Phys. Rev. Lett. 83, 376 (1999).

${ }^{29}$ S. Zhang, Phys. Rev. Lett. 85, 393 (2000).

30 T. Valet and A. Fert, Phys. Rev. B. 48, 7099 (1993).

${ }^{31}$ Q. Yang, P. Holody, S. F. Lee, L. L. Henry, R. Loloee, P. A. Schroeder, W. P. Pratt, Jr., and J. Bass, Phys. Rev. Lett. 72, 3274 (1994).

${ }^{32}$ R. Q. Hood and L. M. Falicov, Phys. Rev. B 46, 8287 (1992).

33 A. Kawabata, Solid State Commun. 34, 431 (1980); A. Kawabata, J. Phys. Soc. Jpn. 49, 628 (1980).

${ }^{34}$ Handbook of Mathematical Functions, edited by M. Abramowitz and I. A. Stegun, Natl. Bur. Stand. (U.S.) Appl. Math. Ser. (GPO, New York, 1964).

${ }^{35}$ L. D. Landau and E. M. Lifshitz, Electrodynamics of Continuous Media (Pergamon, New York, 1984).

${ }^{36}$ B. L. Altshuler and A. G. Aronov, Pis'ma ZhETF 33, 515
(1981) [JETP Lett. 33, 499 (1981)].

${ }^{37}$ V. K. Dugaev and D. E. Khmelnitskii, Zh. Eksp. Teor. Fiz. 86, 1784 (1984) [Sov. Phys. JETP 59, 1038 (1984)].

38 The results of Ref. [ 37 have been obtained in a model of diffuse scattering of electrons on the film boundaries. The non-diffuse scattering can change some numerical constants in Eqs. (80)-(82), see: C. W. J. Beenakker and H. van Houten, Phys. Rev. B 38, 3232 (1988).

${ }^{39}$ M. B. Stearns, in Numerical Data and Functional Relationships in Science and Technology, edited by H. P. J. Wijn, Landolt-Börnstein, New Series, Group III, Vol. 19, Pt. a (Springer, Berlin, 1986).

${ }^{40}$ D. P. Young, D. Hall, M. E. Torrelli, Z. Fisk, J. L. Sarrao, J. D. Thompson, H.-R. Ott, S. B. Oseroff, R. G. Goodrich, and R. Zysler, Nature 397, 412 (1999); H. R. Ott, J. L. Gavilano, B. Ambrosini, V. Vonlanthen, E. Felder, L. Degiorgi, D. P. Young, Z. Fisk, and R. Zysler, Physica B 281-282, 423 (2000). 